\title{
AUTOEXPLORACIÓN DE MAMAS Y SU EJECUCIÓN POR ESTUDIANTES DE MEDICINA
}

\author{
BREAST SELF-EXAMINATION AND ITS PERFORMANCE BY MEDICAL STUDENTS
}

Estudiante de Medicina. Sociedad Cientifica de Estudiantes de Medicina de la Universidad Privada San Juan Bautista (SOCIEM-UPSJB); Universidad Privada San Juan Bautista. Lima, Perú

Estudiante de Medicina. Sociedad Cientifica de Estudiantes de Medicina de la Universidad Continental (SOCEM UC) Universidad Continental. Lima, Perú

Correspondencia a:

Diego André Crisol-Deza

Correo: dcrisoldeza@gmail. com

Telefono: +51988883085

ORCID: https://orcid.org/00000001-7872-3901

https://orcid.org/0000-00017047-0719

palabras clave: Examen Fisico; Neoplasia Inflamatoria de la Mama; Estadisticas de Medicina.

Key words: Physical Examination; Inflammatory Breast Neoplasms; Students, Medical.

Procedencia y arbitraje: comisionado, no sometido a arbitraje.

Recibido para publicación: 25 de abril de 2021

Aceptado para publicación: 12 de junio de 2021

Citar como:

Crisol-Deza DA, Crisol-Deza YG, Autoexploración de mamas y su ejecución por estudiantes de medicina. Rev Cient Cienc Med 2021; 24(1): 204-205

\section{Diego André Crisol-Deza ${ }^{1}$, Yetsli Geraldine Crisol-Deza ${ }^{2}$}

\section{Sr. Editor:}

Según la Organización SMundial de la Salud (OMS), el cáncer de mama es el segundo tumor con mayor prevalencia en el mundo, por lo que es uno de los tumores con mayor morbimortalidad 1. Para ser claramente identificados, los pacientes deben ser monitoreados $y$ controlados anualmente por un profesional médico capacitado, sin embargo, para la autoexploración los pacientes menores de 40 años deben someterse a autoexámenes mensuales y exámenes anuales, mientras que los pacientes entre 40 y 74 años deben iniciar exámenes clínicos de gabinete especialmente mamografías cada 2 a 3 años ${ }^{2}$.

Por otro lado los estudiantes de medicina han jugado un papel muy importante desde que ingresan al campo clínico, en la formación médica y la falta de experiencia puede convertirse en una de las limitaciones de una correcta identificación, se ha observado en los conocimientos prácticos que el $88 \%$ de las estudiantes de medicina mujeres tienen un mejor concepto, a diferencia de los estudiantes de medicina varones ${ }^{3}$. Esto puede deberse a que existen más oportunidades para intercambiar opiniones $y$ experiencias con algún personal médico y / $u$ otras mujeres (círculo familiar $y$ social) sobre el examen de mamas y / o autoexamen, lo que conduce a conocimientos diferenciados entre hombres y mujeres. Según CarrilloLarco $R$ y Col. ${ }^{4}$. en su estudio, el $89,7 \%$ de los estudiantes había oído hablar del autoexamen de mama, pero solo el $21 \%$ de los estudiantes practicaba esta técnica para tener una buena comprensión del cáncer de mama.

Sin embargo, se ha demostrado que en los exámenes de mama ningún resultado positivo es útil para reducir la mortalidad, es decir, el uso de esta técnica no es suficiente para realizar un diagnóstico completo, ya que, un diagnóstico hecho a tiempo, permite actuar inmediatamente para reducir del desarrollo de complicaciones y aumentar la posibilidad de curacion del cáncer, pero si se hace un diagnóstico tardío, la curación completa es mínima, por lo que se deben realizar pruebas auxiliares para obtener un diagnóstico más certero. Según Ramírezy Col${ }^{5}$,el71,1\% de los estudiantes indicaron que el diagnóstico "standar" es una biopsia percutánea. Es importante que los estudiantes $y / o$ profesionales médicos expliquen a las pacientes la importancia del autoexamen como complemento al diagnóstico, y las pacientes que decidan realizarse un autoexamen de mama deben saber que aún no existe evidencia válida de un diagnóstico certero y el riesgo de cáncer de mama es muy alto ${ }^{6}$

Por lo tanto, se recomienda realizar actividades de promoción y prevención para concienciar sobre este problema de salud pública que sigue afectando al siglo $X X I$. Este incremento se puede deber a que aún no hay una capacitación correcta hacia las mujeres sobre la autoexploración y también hacia los estudiantes sobre cómo se debe de realizar una exploración mediante la inspección y palpación, sin embargo, la pandemia de la COVID-19 ha originado mayores deficiencias que limitan la práctica.

El propósito de este trabajo es enfatizar la necesidad urgente de promover la educación clínica y el aprendizaje (educación virtual) a través de esta tecnología, $y$ poder practicar con los círculos familiares y sociales (identificar personas "sin esta enfermedad"), para luego complementarlo en el campo clínico y poder identificarlo, porque dependerá de si se puede reducir este problema que sigue afectando al sistema de salud. Por lo tanto, el método de autoexploración para aplicaciones incorrectas está desactualizado. Existe una necesidad urgente de adquirir los equipos de mamografía más avanzados para mejorar la atención de las pacientes con este tumor maligno y ampliar 
la cobertura del programa de

detección del cáncer.

\section{REFERENCIAS}

1. Organización mundial de la salud. Cáncer de mama: Prevención y control. Disponible: https://WWW. who.int/topics/cancer/breastcancer/ es/

2. Silva-Paredez G, Ruiz BP, Cuba FM. EI autoexamen de mama: Traslación de la evidencia a la práctica clínica. Rev Med Hered [Internet]. 2016 [citado 24 Marzo 2021]; 27:188-189. Disponible: http://www.scielo.org.pe/scielo. php? script $=$ sci arttext\&pid $=S 1018$ $130 \times 2016000300014$

3. Rivero MRJ, Rivero MJ, Bordón $G L$, et al. Factores de riesgo y nivel de conocimientos sobre cáncer de mama en la mujer en estudiantes de Medicina. MediCiego [Internet]. 2020 [citado 24 Marzo 2021];26(1):[aprox. o p.]. Disponible en: http://WwW. revmediciego.s/d.cu/index.php/ mediciego/article/view/1808.

4. Carrillo-Larco RM., EspinozaSalguero ME., Avilez J, et al. Nivel de conocimiento $y$ frecuencia de autoexamen de mama en alumnos de los primeros años de la carrera de Medicina. Rev Med Hered [Internet]. 2015 Oct [citado $2021 \mathrm{Mar}$ 23];26(4):209-216. Disponible en: http://www.scielo.org.pe/pdf/rmh/ v26n4/a02v26n4.pdf

5. Santibáñez Ramírez Meybül, Símbala Delgado Andrea, Valenzuela Núñez Nathalie, Morales Ojeda Ismael, Gelabert Santané Ramón.
Conocimiento del cáncer de mama en estudiantes de enfermerla. Cienc. enferm. [Internet]. 2019 [citado 2021 Mar 24];25: Disponible en: https://scielo.conicyt.cl/scielo. php?script=sci_arttext\&pid=S071795532019000100205\&/ng=es

6. Sánchez UY, Urdaneta MJR, Villalobos IN, Contreras BA, García J, Baabel ZN, et al. Conocimiento sobre cáncer de mamas y práctica del autoexamen de mamas en mujeres de edad mediana. Revista Venezolana de Oncología [Internet]. 2016 [citado $2021 \mathrm{Jul}$ 15].28(1):3751. Disponible: https://WWW.redalyc. org/jatsRepo/3756/375643222006/ html/index.htm/ 